\title{
A Carcinus with a right-handed walking-leg on the left side of the abdomen.
}

\author{
PRELIMINARY NOTE.
}

By

Albrecht Bethe.

Amongst a great number of crabs collected in Plymouth Sound for my studies of the central nervous system, one specimen was found with a very interesting abnormality. The thorax of the crab-a female-is normal. The length of the carapace is $47 \mathrm{~mm}$., the breadth $64 \mathrm{~mm}$. The claws and legs are in the right positions, and of normal proportions, with the exception of the fourth leg on the left side, which is smaller than usual. In my opinion, this leg was broken some time before the crab came in, and was not yet quite regenerated. The first four segments of the abdomen are also normal. Each has a couple of pedes spurii. To the fifth segment, which in the crab is always legless, a large leg is fastened on the left side. (Fig. 1.)

This leg is, as one can see at a glance, a real walking-leg, and, what may at first sight seem strange, not a left-handed, but a right-handed leg. Comparing it with the other walking-legs, one can see that this additional leg corresponds to the second and third of the thorax. Not only are the positions of the hairs and little pits the same as in those legs, but also the proportions between the single joints and the angles which form the axes of the joints.

Owing to the pressure of the large leg, the left side of the fifth abdominal segment is a little bent on both sides, and the exopodite of the left pes spurius of the fourth segment is stunted, so that it is not half as long as the exopodite of the other side. When alive, the leg was motionless, but it was sensitive.

When first I saw the crab, I imagined this surplus leg must be innervated by a nerve coming from a surplus half-ganglion of the right side. And this indeed proved to be the case. A dissection made 
from the back showed a big nerve entering the leg, and starting from the right side of the ventral cord. But, strange to say, the nerve does not leave the ventral cord at its side, but in the middle, passing through the middle hole. I cut thick frontal sections of the ventral cord. They show a small surplus half-ganglion on the right side, between the claw-ganglion and the ganglion of the first walking-leg,

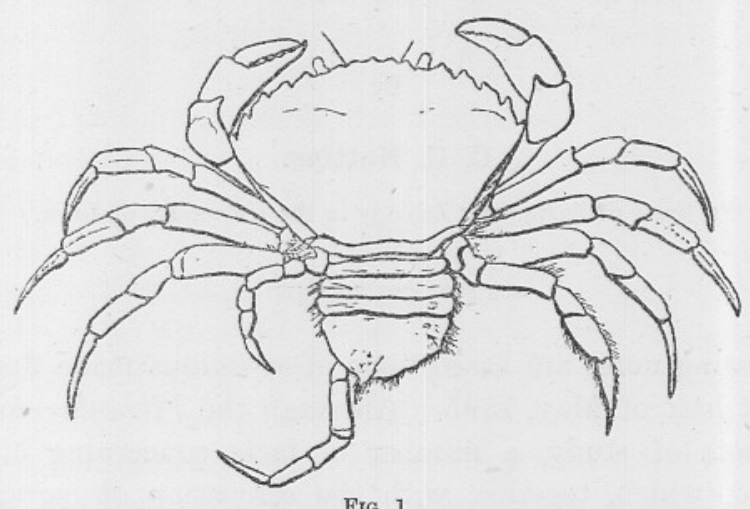

FIG. 1.

and not, as was to be expected, between the first and second or the second and third leg-ganglion.

There is no doubt that in this case we have neither a double-monster nor an atavism, because the ancestors of the Brachyura never had walking-legs on the abdomen.

A second paper on this subject, containing minute descriptions and several illustrations, will soon be published in a German journal, and in that I will endeavour to give a theoretical explanation of this abnormality. 